\title{
REFINEMENTS AND REVERSES OF HöLDER-MCCARTHY OPERATOR INEQUALITY VIA A CARTWRIGHT-FIELD RESULT
}

\author{
Silvestru Sever Dragomir
}

\begin{abstract}
(C) by University of Niš, Serbia | Creative Commons Licence: CC BY-NC-ND Abstract. By the use of a classical result of Cartwright and Field, in this paper we have obtained new refinements and reverses of Hölder-McCarthy operator inequality in the case of $p \in(0,1)$. A comparison for the two upper bounds obtained showing that neither of them is better in general, has also been performed.

Keywords: Hölder-McCarthy operator inequality; selfadjoint operator; Hilbert space; nonnegative operator.
\end{abstract}

\section{Introduction}

Let $A$ be a nonnegative operator on the complex Hilbert space $(H,\langle\cdot, \cdot\rangle)$, namely $\langle A x, x\rangle \geq 0$ for any $x \in H$. We write this as $A \geq 0$.

By the use of the spectral resolution of $A$ and the Hölder inequality, C. A. McCarthy [16] proved that

$$
\langle A x, x\rangle^{p} \leq\left\langle A^{p} x, x\right\rangle, p \in(1, \infty)
$$

and

$$
\left\langle A^{p} x, x\right\rangle \leq\langle A x, x\rangle^{p}, p \in(0,1)
$$

for any $x \in H$ with $\|x\|=1$.

Let $A$ be a selfadjoint operator on $H$ with

$$
m I \leq A \leq M I
$$

where $I$ is the identity operator and $m, M$ are real numbers with $m<M$.

Received September 30, 2019; accepted February 25, 2020

2010 Mathematics Subject Classification. Primary 47A63, 47A30; Secondary 15A60, 26D15; 26D10 
In [7, Theorem 3] Fujii et al. obtained the following interesting ratio inequality that provides a reverse of the Hölder-McCarthy inequality (1.1) for an operator $A$ that satisfies the condition (1.3) with $m>0$

$$
\left\langle A^{p} x, x\right\rangle \leq\left\{\frac{1}{p^{1 / p} q^{1 / q}} \frac{M^{p}-m^{p}}{(M-m)^{1 / p}\left(m M^{p}-M m^{p}\right)^{1 / q}}\right\}^{p}\langle A x, x\rangle^{p},
$$

for any $x \in H$ with $\|x\|=1$, where $q=p /(p-1), p>1$.

If $A$ satisfies the condition (1.3) with $m \geq 0$, then we also have the additive reverse of (1.1) that has been obtained by the author in 2008, see [4]

$$
\begin{gathered}
\left\langle A^{p} x, x\right\rangle-\langle A x, x\rangle^{p} \leq \frac{1}{2} p(M-m)\left[\left\|A^{p-1} x\right\|^{2}-\left\langle A^{p-1} x, x\right\rangle^{2}\right]^{1 / 2} \\
\leq \frac{1}{4} p(M-m)\left(M^{p-1}-m^{p-1}\right)
\end{gathered}
$$

and

$$
\begin{gathered}
\left\langle A^{p} x, x\right\rangle-\langle A x, x\rangle^{p} \leq \frac{1}{2} p\left(M^{p-1}-m^{p-1}\right)\left[\|A x\|^{2}-\langle A x, x\rangle^{2}\right]^{1 / 2} \\
\leq \frac{1}{4} p(M-m)\left(M^{p-1}-m^{p-1}\right)
\end{gathered}
$$

for any $x \in H$ with $\|x\|=1$, where $p>1$.

We also have the alternative upper bounds [4]

$$
\begin{aligned}
\left\langle A^{p} x, x\right\rangle-\langle A x, x\rangle^{p} \leq & \frac{1}{4} p \frac{(M-m)\left(M^{p-1}-m^{p-1}\right)}{M^{p / 2} m^{p / 2}}\langle A x, x\rangle\left\langle A^{p-1} x, x\right\rangle, \quad(\text { for } m>0) \\
& \leq p \frac{1}{4}(M-m)\left(M^{p-1}-m^{p-1}\right)\left(\frac{M}{m}\right)^{p / 2},(\text { for } m>0)
\end{aligned}
$$

and

$$
\begin{gathered}
\left\langle A^{p} x, x\right\rangle-\langle A x, x\rangle^{p} \leq p(\sqrt{M}-\sqrt{m})\left(M^{(p-1) / 2}-m^{(p-1) / 2}\right)\left[\langle A x, x\rangle\left\langle A^{p-1} x, x\right\rangle\right]^{\frac{1}{2}} \\
\leq p(\sqrt{M}-\sqrt{m})\left(M^{(p-1) / 2}-m^{(p-1) / 2}\right) M^{p / 2}
\end{gathered}
$$

for any $x \in H$ with $\|x\|=1$, where $p>1$.

For various related inequalities, see [6]-[10] and [14]-[15].

We have the following inequality that provides a refinement and a reverse for the celebrated Young's scalar inequality

$$
\begin{aligned}
\frac{1}{2} \nu(1-\nu) \frac{(b-a)^{2}}{\max \{a, b\}} & \leq(1-\nu) a+\nu b-a^{1-\nu} b^{\nu} \\
& \leq \frac{1}{2} \nu(1-\nu) \frac{(b-a)^{2}}{\min \{a, b\}}
\end{aligned}
$$

for any $a, b>0$ and $\nu \in[0,1]$. 
This result was obtained in 1978 by Cartwright and Field [1] who established a more general result for $n$ variables and gave an application for a probability measure supported on a finite interval.

For new recent reverses and refinements of Young's inequality see [2]-[3], [11][12], [13] and [19].

By the use of (1.5). we have obtained new refinements and reverses of HölderMcCarthy operator inequality in the case when $p \in(0,1)$. A comparison for the two upper bounds obtained showing that neither of them is better in general, has also been performed.

\section{Some Refinements and Reverse Results}

We have:

Theorem 2.1. Let $m, M$ be real numbers so that $M>m>0$. If $A$ is a selfadjoint operator satisfying the condition (1.3) above, then for any $p \in(0,1)$ we have

$$
\begin{array}{r}
\frac{p(1-p)}{2} \frac{m}{M}\left(\frac{\left\langle A^{2} x, x\right\rangle}{\langle A x, x\rangle^{2}}-1\right) \leq \frac{p(1-p)}{2 M}\langle A x, x\rangle\left(\frac{\left\langle A^{2} x, x\right\rangle}{\langle A x, x\rangle^{2}}-1\right) \\
\leq 1-\frac{\left\langle A^{p} x, x\right\rangle}{\langle A x, x\rangle^{p}} \\
\leq \frac{p(1-p)}{2 m}\langle A x, x\rangle\left(\frac{\left\langle A^{2} x, x\right\rangle}{\langle A x, x\rangle^{2}}-1\right) \\
\leq \frac{p(1-p)}{2} \frac{M}{m}\left(\frac{\left\langle A^{2} x, x\right\rangle}{\langle A x, x\rangle^{2}}-1\right)
\end{array}
$$

for any $x \in H$ with $\|x\|=1$.

In particular,

$$
\begin{gathered}
\frac{1}{8} \frac{m}{M}\left(\frac{\left\langle A^{2} x, x\right\rangle}{\langle A x, x\rangle^{2}}-1\right) \leq \frac{\langle A x, x\rangle}{8 M}\left(\frac{\left\langle A^{2} x, x\right\rangle}{\langle A x, x\rangle^{2}}-1\right) \\
\leq 1-\frac{\left\langle A^{1 / 2} x, x\right\rangle}{\langle A x, x\rangle^{1 / 2}} \leq \frac{\langle A x, x\rangle}{8 m}\left(\frac{\left\langle A^{2} x, x\right\rangle}{\langle A x, x\rangle^{2}}-1\right) \\
\leq \frac{1}{8} \frac{M}{m}\left(\frac{\left\langle A^{2} x, x\right\rangle}{\langle A x, x\rangle^{2}}-1\right),
\end{gathered}
$$

for any $x \in H$ with $\|x\|=1$.

Proof. If $a, b \in[m, M]$, then by Cartwright-Field inequality (1.5) we have

$$
\frac{1}{2 M} p(1-p)(b-a)^{2} \leq(1-p) a+p b-a^{1-p} b^{p} \leq \frac{1}{2 m} p(1-p)(b-a)^{2}
$$


or, equivalently

$$
\begin{aligned}
\frac{1}{2 M} p(1-p)\left(b^{2}-2 a b+a^{2}\right) & \leq(1-p) a+p b-a^{1-p} b^{p} \\
& \leq \frac{1}{2 m} p(1-p)\left(b^{2}-2 a b+a^{2}\right)
\end{aligned}
$$

for any $p \in(0,1)$.

Fix $a \in[m, M]$ and by using the operator functional calculus for $A$ with $m I \leq$ $A \leq M I$ we have

$$
\begin{aligned}
\frac{1}{2 M} p(1-p)\left(A^{2}-2 a A+a^{2} I\right) & \leq(1-p) a I+p A-a^{1-p} A^{p} \\
& \leq \frac{1}{2 m} p(1-p)\left(A^{2}-2 a A+a^{2} I\right)
\end{aligned}
$$

Then for any $x \in H$ with $\|x\|=1$ we have from (2.4) that

$$
\begin{aligned}
& \frac{1}{2 M} p(1-p)\left(\left\langle A^{2} x, x\right\rangle-2 a\langle A x, x\rangle+a^{2}\right) \\
& \leq(1-p) a+p\langle A x, x\rangle-a^{1-p}\left\langle A^{p} x, x\right\rangle \\
\leq & \frac{1}{2 m} p(1-p)\left(\left\langle A^{2} x, x\right\rangle-2 a\langle A x, x\rangle+a^{2}\right),
\end{aligned}
$$

for any $a \in[m, M]$.

If we choose in (2.5) $a=\langle A x, x\rangle \in[m, M]$, then we get for any $x \in H$ with $\|x\|=1$ that

$$
\begin{aligned}
\frac{1}{2 M} p(1-p)\left(\left\langle A^{2} x, x\right\rangle-\langle A x, x\rangle^{2}\right) & \leq\langle A x, x\rangle-\langle A x, x\rangle^{1-p}\left\langle A^{p} x, x\right\rangle \\
\leq & \frac{1}{2 m} p(1-p)\left(\left\langle A^{2} x, x\right\rangle-\langle A x, x\rangle^{2}\right)
\end{aligned}
$$

and by division with $\langle A x, x\rangle>0$ we obtain the second and third inequalities in $(2.1)$.

The rest is obvious.

Remark 2.1. It is well known that, if $m I \leq A \leq M I$ with $M>0$, then, see for instance $[17$, p. 27], we have

$$
(1 \leq) \frac{\left\langle A^{2} x, x\right\rangle}{\langle A x, x\rangle^{2}} \leq \frac{(m+M)^{2}}{4 m M}
$$

for any $x \in H$ with $\|x\|=1$, which implies that

$$
(0 \leq) \frac{\left\langle A^{2} x, x\right\rangle}{\langle A x, x\rangle^{2}}-1 \leq \frac{(M-m)^{2}}{4 m M} .
$$

Using (2.1) and by denoting $h=\frac{M}{m}$ we get

$$
(0 \leq) 1-\frac{\left\langle A^{p} x, x\right\rangle}{\langle A x, x\rangle^{p}} \leq \frac{p(1-p)}{8}(h-1)^{2}
$$


and, in particular,

$$
(0 \leq) 1-\frac{\left\langle A^{1 / 2} x, x\right\rangle}{\langle A x, x\rangle^{1 / 2}} \leq \frac{1}{32}(h-1)^{2}
$$

for any $x \in H$ with $\|x\|=1$.

We consider the Kantorovich's constant defined by

$$
K(h):=\frac{(h+1)^{2}}{4 h}, h>0 .
$$

The function $K$ is decreasing on $(0,1)$ and increasing on $[1, \infty), K(h) \geq 1$ for any $h>0$ and $K(h)=K\left(\frac{1}{h}\right)$ for any $h>0$.

Observe that for any $h>0$

$$
K(h)-1=\frac{(h-1)^{2}}{4 h}=K\left(\frac{1}{h}\right)-1 .
$$

From (2.6) we then have

$$
(0 \leq) 1-\frac{\left\langle A^{p} x, x\right\rangle}{\langle A x, x\rangle^{p}} \leq \frac{p(1-p)}{2} h[K(h)-1]
$$

and, in particular,

$$
(0 \leq) 1-\frac{\left\langle A^{1 / 2} x, x\right\rangle}{\langle A x, x\rangle^{1 / 2}} \leq \frac{1}{8} h[K(h)-1],
$$

for any $x \in H$ with $\|x\|=1$.

Also, if $a, b>0$ then

$$
K\left(\frac{b}{a}\right)-1=\frac{(b-a)^{2}}{4 a b} .
$$

Since $\min \{a, b\} \max \{a, b\}=a b$ if $a, b>0$, then

$$
\frac{(b-a)^{2}}{\max \{a, b\}}=\frac{\min \{a, b\}(b-a)^{2}}{a b}=4 \min \{a, b\}\left[K\left(\frac{b}{a}\right)-1\right]
$$

and

$$
\frac{(b-a)^{2}}{\min \{a, b\}}=\frac{\max \{a, b\}(b-a)^{2}}{a b}=4 \max \{a, b\}\left[K\left(\frac{b}{a}\right)-1\right]
$$

and the inequality (1.5) can be written as

$$
\begin{aligned}
& 2 \nu(1-\nu) \min \{a, b\}\left[K\left(\frac{b}{a}\right)-1\right] \leq(1-\nu) a+\nu b-a^{1-\nu} b^{\nu} \\
& \leq 2 \nu(1-\nu) \max \{a, b\}\left[K\left(\frac{b}{a}\right)-1\right]
\end{aligned}
$$

for any $a, b>0$ and $\nu \in[0,1]$. 
Theorem 2.2. Let $m, M$ be real numbers so that $M>m>0$. If $A$ is a selfadjoint operator satisfying the condition (1.3) above, then for any $p \in(0,1)$ we have

$$
\begin{gathered}
(0 \leq) 1-\frac{\left\langle A^{p} x, x\right\rangle}{\langle A x, x\rangle^{p}} \\
\leq p(1-p)[K(h)-1]\left(2+\frac{\langle|A-\langle A x, x\rangle I| x, x\rangle}{\langle A x, x\rangle}\right) \\
\leq p(1-p)[K(h)-1]\left[2+\left(\frac{\left\langle A^{2} x, x\right\rangle}{\langle A x, x\rangle^{2}}-1\right)^{1 / 2}\right] \\
\leq p(1-p)[K(h)-1]\left[2+(K(h)-1)^{1 / 2}\right]
\end{gathered}
$$

for any $x \in H$ with $\|x\|=1$.

In particular, we have

$$
\begin{gathered}
(0 \leq) 1-\frac{\left\langle A^{1 / 2} x, x\right\rangle}{\langle A x, x\rangle^{1 / 2}} \\
\leq \frac{1}{4}[K(h)-1]\left(2+\frac{\langle|A-\langle A x, x\rangle I| x, x\rangle}{\langle A x, x\rangle}\right) \\
\leq \frac{1}{4}[K(h)-1]\left[2+\left(\frac{\left\langle A^{2} x, x\right\rangle}{\langle A x, x\rangle^{2}}-1\right)^{1 / 2}\right] \\
\leq \frac{1}{4}[K(h)-1]\left[2+(K(h)-1)^{1 / 2}\right]
\end{gathered}
$$

for any $x \in H$ with $\|x\|=1$.

Proof. From (2.11) we have for any $a, b>0$ and $p \in[0,1]$ that

$$
(1-p) a+p b-a^{1-p} b^{p} \leq p(1-p)(a+b+|b-a|)\left[K\left(\frac{b}{a}\right)-1\right]
$$

since

$$
\max \{a, b\}=\frac{1}{2}(a+b+|b-a|) .
$$

If $a, b \in[m, M]$, then $\frac{b}{a} \in\left[\frac{m}{M}, \frac{M}{m}\right]$ and by the properties of Kantorovich's constant $K$, we have

$$
1 \leq K\left(\frac{b}{a}\right) \leq K\left(\frac{M}{m}\right)=K(h) \text { for any } a, b \in[m, M] .
$$

Therefore, by (2.13) we have

$$
(1-p) a+p b-a^{1-p} b^{p} \leq p(1-p)(a+b+|b-a|)[K(h)-1]
$$

for any $a, b \in[m, M]$ and $p \in[0,1]$.

Fix $a \in[m, M]$ and by using the operator functional calculus for $A$ with $m I \leq$ $A \leq M I$, we have

$$
(1-p) a I+p A-a^{1-p} A^{p} \leq p(1-p)[K(h)-1](a I+A+|A-a I|) .
$$


Then for any $x \in H$ with $\|x\|=1$ we get from (2.14) that

$$
\begin{gathered}
(1-p) a+p\langle A x, x\rangle-a^{1-p}\left\langle A^{p} x, x\right\rangle \\
\leq p(1-p)[K(h)-1](a+\langle A x, x\rangle+\langle|A-a I| x, x\rangle),
\end{gathered}
$$

for any $a \in[m, M]$ and $p \in[0,1]$.

Now, if we take $a=\langle A x, x\rangle \in[m, M]$, where $x \in H$ with $\|x\|=1$ in (2.15), then we obtain

$$
\begin{gathered}
\langle A x, x\rangle-\langle A x, x\rangle^{1-p}\left\langle A^{p} x, x\right\rangle \\
\leq p(1-p)[K(h)-1](2\langle A x, x\rangle+\langle|A-\langle A x, x\rangle I| x, x\rangle),
\end{gathered}
$$

which, by division with $\langle A x, x\rangle>0$ provides the first inequality in (2.11).

By Schwarz inequality, we have for $x \in H$ with $\|x\|=1$ that

$$
\begin{aligned}
\langle|A-\langle A x, x\rangle I| x, x\rangle & \leq\left\langle(A-\langle A x, x\rangle I)^{2} x, x\right\rangle^{1 / 2} \\
& =\left\langle\left(A^{2}-2\langle A x, x\rangle A+\langle A x, x\rangle^{2} I\right) x, x\right\rangle^{1 / 2} \\
& =\left(\left\langle A^{2} x, x\right\rangle-\langle A x, x\rangle^{2}\right)^{1 / 2}
\end{aligned}
$$

which proves the second part of (2.11).

Since

$$
\frac{\left\langle A^{2} x, x\right\rangle}{\langle A x, x\rangle^{2}}-1 \leq \frac{(M-m)^{2}}{4 m M}=K(h)-1
$$

for $x \in H$ with $\|x\|=1$, then the last part of (2.11) is thus proved.

\section{A Comparison for Upper Bounds}

We observe that the inequality (2.9) provides for the quantity

$$
(0 \leq) 1-\frac{\left\langle A^{p} x, x\right\rangle}{\langle A x, x\rangle^{p}}, x \in H \text { with }\|x\|=1,
$$

the following upper bound

$$
B_{1}(p, h):=\frac{p(1-p)}{2} h[K(h)-1],
$$

while the inequality (2.11) gives the upper bound

$$
B_{2}(p, h):=p(1-p)[K(h)-1]\left[2+(K(h)-1)^{1 / 2}\right],
$$

where $p \in(0,1)$ and $h>1$. 
Now, if we depict the 3D plot for the difference of the bounds $B_{1}$ and $B_{2}$, namely

$$
D(x, y):=B_{1}(y, x)-B_{2}(y, x)
$$

on the box $[1,8] \times[0,1]$, then we observe that it takes both positive and negative values, showing that the bounds $B_{1}(p, h)$ and $B_{2}(p, h)$ can not be compared in general, namely neither of them is better for any $p \in(0,1)$ and $h>1$.

\section{R E F E R E N C E S}

1. D. I. CARtwright, M. J. Field: A refinement of the arithmetic mean-geometric mean inequality, Proc. Amer. Math. Soc., 71 (1978), 36-38.

2. S. S. Dragomir: A note on Young's inequality, Preprint RGMIA Res. Rep. Coll. 18 (2015), Art. 126. [http://rgmia.org/papers/v18/v18a126.pdf] .

3. S. S. Dragomir: A note on new refinements and reverses of Young's inequality, Preprint RGMIA Res. Rep. Coll. 18 (2015), Art. . [http://rgmia.org/papers/v1] .

4. S. S. Dragomir: Some reverses of the Jensen inequality for functions of selfadjoint operators in Hilbert spaces, J. Inequal. \& Appl., Volume 2010, Article ID 496821, 15 pages doi:10.1155/2010/496821. Preprint RGMIA Res. Rep. Coll., 11 (2008), Supliment. Art. 15. [Online http://rgmia.org/papers/v11e/RevJensenOp.pdf] .

5. S. S. Dragomir: Operator Inequalities of the Jensen, Čebyšev and Grüss Type, Springer Briefs in Mathematics, Springer, 2012.

6. M. Fujin, S. Izumino, R. Nakamoto: Classes of operators determined by the HeinzKato-Furuta inequality and the Hölder-McCarthy inequality. Nihonkai Math. J. 5 (1994), no. 1, 61-67.

7. M. FujiI, S. Izumino, R. Nakamoto, Y. Seo: Operator inequalities related to Cauchy-Schwarz and Hölder-McCarthy inequalities, Nihonkai Math. J. 8 (1997), 117122.

8. T. Furuta: Extensions of Hölder-McCarthy and Kantorovich inequalities and their applications. Proc. Japan Acad. Ser. A Math. Sci. 73 (1997), no. 3, 38-41.

9. T. FuRutA: Operator inequalities associated with Hölder-McCarthy and Kantorovich inequalities. J. Inequal. Appl. 2 (1998), no. 2, 137-148.

10. T. FurutA: The Hölder-McCarthy and the Young inequalities are equivalent for Hilbert space operators. Amer. Math. Monthly 108 (2001), no. 1, 68-69.

11. F. Kittaneh, Y. Manasrah: Improved Young and Heinz inequalities for matrix, J. Math. Anal. Appl. 361 (2010), 262-269.

12. F. Kittaneh, Y. Manasrah: Reverse Young and Heinz inequalities for matrices, Linear Multilinear Algebra, 59 (2011), 1031-1037.

13. W. LiaO, J. Wu, J. ZhaO: New versions of reverse Young and Heinz mean inequalities with the Kantorovich constant, Taiwanese J. Math. 19 (2015), No. 2, pp. 467-479.

14. C.-S. Lin, Y. J. Сно: On Hölder-McCarthy-type inequalities with powers. J. Korean Math. Soc. 39 (2002), no. 3, 351-361. 
15. C.-S. Lin A, Y. J. CHO: On Kantorovich inequality and Hölder-McCarthy inequalities. Dyn. Contin. Discrete Impuls. Syst. Ser. A Math. Anal. 11 (2004), no. 4, 481-490.

16. C. A. MCCARThY: $c_{p}$, Israel J. Math. 5 (1967), 249-271.

17. J. PeČarić, T. Furuta, J. Mićić Hot, Y. SeO: Mond-Pečarić Method in Operator inequalities. Inequalities for Bounded Selfadjoint Operators on a Hilbert Space. Monographs in Inequalities, 1. ELEMENT, Zagreb, 2005. xiv+262 pp.+loose errata. ISBN: 953-197-572-8

18. W. Specht: Zer Theorie der elementaren Mittel, Math. Z., 74 (1960), pp. 91-98.

19. M. Tominaga: Specht's ratio in the Young inequality, Sci. Math. Japon., 55 (2002), 583-588.

Silvestru Sever Dragomir

College of Engineering and of Science

Department of Mathematics

P. O. Box 8000

Melbourne, Australia

sever.dragomir@vu.edu.au 